\title{
Model-based shading and lighting controls considering visual comfort and energy use
}

\author{
Jie Xiong ${ }^{\mathrm{a}, \mathrm{b}}$, Athanasios Tzempelikos ${ }^{\mathrm{a}, \mathrm{b}, *}$ \\ ${ }^{a}$ Lyles School of Civil Engineering, Purdue University, 550 Stadium Mall Dr., West Lafayette, Indiana, \\ 47907, USA \\ ${ }^{b}$ Center for High Performance Buildings, Ray W. Herrick Laboratories, 140 S. Martin Jischke Dr., West \\ Lafayette, Indiana, 47907, USA
}

\begin{abstract}
Dynamic facades with high performance glazing and shading systems have the potential to balance daylighting needs, comfort and energy use, when integrated with lighting and thermal system controls. This paper presents the development and implementation of model-based control (MBC) algorithms for shading and lighting operation, aiming at minimizing lighting energy use while satisfying glare constraints. A fast and reliable lighting-glare model was used to compute real-time interior lighting conditions, lighting energy use and daylight glare probability, for predetermined shade positions (10\% increments) at each control time step, based on the readings of two sensors on each building facade. Three visual comfort criteria (DGP, vertical and work plane illuminance) were embedded and compared in the real-time shading control logic, which selects the highest shade position (maximum daylight utilization) that satisfies the visual comfort criteria at each time step. The approach is similar to an optimization scheme for discrete shade positions, using visual comfort constraints as a priority, but here it is applied efficiently with a semi-analytical model in real-time control.

The developed MBC strategies were successfully demonstrated in office spaces, controlling shades and electric lighting in real-time, using simple sensor readings as inputs. An innovative, variable control interval logic was also developed and implemented, resulting in reduced shading operation without sacrificing the benefits, creating less disturbance for occupants and extending equipment life. Finally, the developed MBC strategies were evaluated using annual simulation, in terms of frequency of shade movements, annual percentage of working hours with risk of glare, lighting energy use, and fraction of outside view.
\end{abstract}

Keywords: model-based control, shading, lighting, visual comfort, energy

\section{Introduction}

Energy efficiency and human comfort in buildings are the top priorities in building design and operation. Commercial buildings in the United States consumed 5.27.10 $0^{12} \mathrm{kWh}$ of energy in 2013 (EIA 2015). More than one third of the electricity consumed in commercial buildings is due to electric lighting. Therefore, reducing lighting energy use is a major goal towards overall energy efficiency in buildings.

Properly designed and controlled fenestration systems, coordinated with lighting operation, should provide natural light while ensuring occupant comfort under changing weather conditions, reducing electric lighting demand and energy use. To evaluate the impact of advanced control strategies, accurate and efficient models of dynamic façade and lighting systems are needed, as well as proper comfort indices, for use in smart automatic control algorithms (Lindelof, 2009) and overall strategies. Model-Based Control (MBC) strategies that use (detailed or low-order) models with real-time input data and robust control schemes have a great potential to reduce energy use and improve indoor environmental comfort. Most of the studies in this field have focused on thermal/HVAC system operation (Li et al., 2014, Morosan et al., 2010, Privara et al., 2011, Derakhtenjani et al., 2015, Cai et al., 2015) and indoor air quality control applications ( $\mathrm{Lu}$ et al, 2011), with embedded optimization techniques (Li et al., 2015, Hu and Karava, 2014).

*Corresponding author. Tel.: +765-496-7586.

E-mail address: ttzempel@purdue.edu (A. Tzempelikos)

(C) 2016. This manuscript version is made available under the Elsevier user license

http://www.elsevier.com/open-access/userlicense/1.0/ 
However, only a few studies applied MBC strategies for lighting and shading controls (Le et al, 2014, Grube et al., 2014), initiated by Mahdavi (2008). Thorough work on blind controllers with multi-objective optimization processes and extensions to user wishes (Daum and Morel, 2010, Guillemin and Molteni, 2002) provide advanced and promising solutions. Fischer et al. (2012) utilized an accurate model trained by measured data from light sensors located at every seat for multi-preference electric lighting control. Kim and Park (2012) used EnergyPlus as a model-based predictor to obtain optimal slat angles within a $24 \mathrm{hr}$ time horizon -with high computational effort. Shen et al. (2014) studied independent open and closed loop strategies for shading and lighting, as well as integrated controls including temperature and occupancy information. Recently, Bueno et al. (2015) developed a new Radiance-based modeling approach (Fener) for analysis and control of complex fenestration systems, which couples daylighting and thermal modeling in a time-step basis. This model, which uses BSDF data and the three-phase method, can be used to implement advanced shading control algorithms.

Very few studies directly associated visual comfort or glare indices with shading controls (Oh et al., 2012), although daylight glare is one of the primary reasons for interacting with shading systems. Wienold (2007) used the simplified Daylight Glare Probability approximation (DGPs) to evaluate the efficiency of shading controls towards glare -however, the strategies were not based on glare but only evaluated in terms of it. Similarly, Yun et al. (2014) used DGP to evaluate blind control strategies towards glare and energy and first stated that $E_{v}$ is a good criterion for shading control. However, from their correlations with DGP and DGPs, it is implied that no direct light conditions were met. Obtaining real-time DGP data is challenging. As the DGPs approximation uses only vertical illuminance, which can be measured or simulated in real time, the potential of a model-based control based on DGPs is promising (Konstantzos et al., 2015).

Recent MBC studies on shading and lighting require real-time detailed simulation and extensive sensor networks for acquiring necessary information with changing weather and sky conditions. In addition, improper or separate controls for façade and lighting systems could be ineffective and costly. Therefore a low-cost but reliable integrated shading and lighting model-based control, with less exogenous inputs is desired. In that way, model-based control algorithms could be effective in management of façade, interior lighting and comfort (Mahdavi et al., 2008). Moreover, previous studies focused on venetian blinds; application to roller shades is quite limited, although simple and advanced heuristic control rules have been developed (Tzempelikos and Shen, 2013).

This paper presents the development and implementation of shading and lighting model-based control algorithms, based on visual comfort criteria, for perimeter zones with interior roller shades. The control strategies were implemented in full-scale offices and were able to minimize lighting energy use while maintaining visual comfort. Advanced control options that consider variable control intervals were developed and implemented. Finally, applications in annual building simulation are discussed.

\section{Integrated shading and lighting model-based control}

The flowchart of the developed integrated model-based control (MBC) is shown in Fig. 1. The logic consists of the following steps. Details on implementation and device controls are presented in a later section.

\subsection{Model inputs and sensor readings}

Beam and diffuse (sky and ground-reflected) transmitted illuminance through the window (calculated or measured by sensors for real-time control, or TMY3 data for an annual analysis) are required as inputs to the daylighting and glare simulation model. At each time step, these quantities are obtained by processing the readings of two exterior sensors (global horizontal illuminance and direct-diffuse ratio of incident solar radiation on the façade) and one interior sensor (total transmitted illuminance). Note that alternate calculation methods could eliminate the need for the horizontal illuminance sensor, although only one such sensor is needed for each location. More detailed measurements of sky and ground-reflected illuminance would require the use of extra sensors, therefore increasing sensor network complexity.

\subsection{Daylight and glare simulation model for discrete shade positions}

Space geometry, fenestration system optical properties - easily obtained from WINDOW software (LBNL, 2014) - and occupancy information (seating location and viewing direction) are pre-determined 
parameters, also inputs to the model. Together with current local time, these are used to calculate interior illuminance and luminance distributions based on a validated hybrid ray-tracing and radiosity daylight model (Chan and Tzempelikos, 2012). The model combines the accuracy of forward ray tracing for direct light with the computational efficiency of radiosity for diffuse light entering the space and interior reflections. Angular direct-direct and direct-diffuse light transmission through roller shades is calculated using the semi-empirical model by Kotey et al. (2009), which has been validated using integrated sphere measurements and full-scale experiments (Chan et al., 2015). Other models can be used for different types of complex fenestration systems, but this model is simple, analytical and accurate (i.e., fast and easy to implement in real-time control).

Each interior surface is discretized into sub-surfaces (for increased accuracy) and the lighting model outputs include work plane illuminance, vertical (on eye) illuminance, and DGP for a pre-selected calculation grid of virtual sensors (occupant positions and view directions), based on the full DGP equation (Wienold and Christoffersen, 2006):

$D G P=5.87 \times 10^{-5} E_{v}+9.18 \times 10^{-2} \log _{10}\left(1+\sum_{i}^{n} \frac{L_{S, i}{ }^{2} \omega_{s, i}}{E_{v}{ }^{1.87} P_{i}{ }^{2}}\right)+0.16$

where $L_{s}$ is glare source luminance, $\omega_{s}$ is source solid angle, $P$ is the position index and $E_{v}$ is the vertical illuminance on the eye. Real-time DGP calculation is challenging, but the model efficiency (in open source language) allows calculation of luminance distributions (from illuminance values) almost instantaneously, which maintains the efficiency of the developed model-based control. The position index used in the calculation is a combination of Guth's model (Guth 1966) and Inoue's model (Inoue et al. 1988). Position indices, solid angles, and the angles between the line of sight and the lines connecting the eye and the patches of every surface are all pre-calculated. Patches with angles higher than $78^{\circ}$ are outside the occupant's field of view and are neglected. For patches inside the field of view, the luminance values are compared to a reference value to determine if the patch is a glare source. In this paper, the reference glare threshold is defined as 4 times of the average work plane luminance, as suggested by Wienold (2012). Other threshold criteria are also possible and easy to implement (Van Den Wymelenberg and Inanici, 2014).

To ensure computational efficiency and responsive speed for real-time control, the hybrid daylightingglare model calculates all the outputs for 11 pre-defined, discrete shading positions ( $10 \%$ increments), from fully open to fully closed position, at each time step (1 minute in this study). This step is critical for fast processing of results and enables simpler control decisions (moves shades to the selected intermediate position). The set of simulation outputs are then sent to a decision maker. 


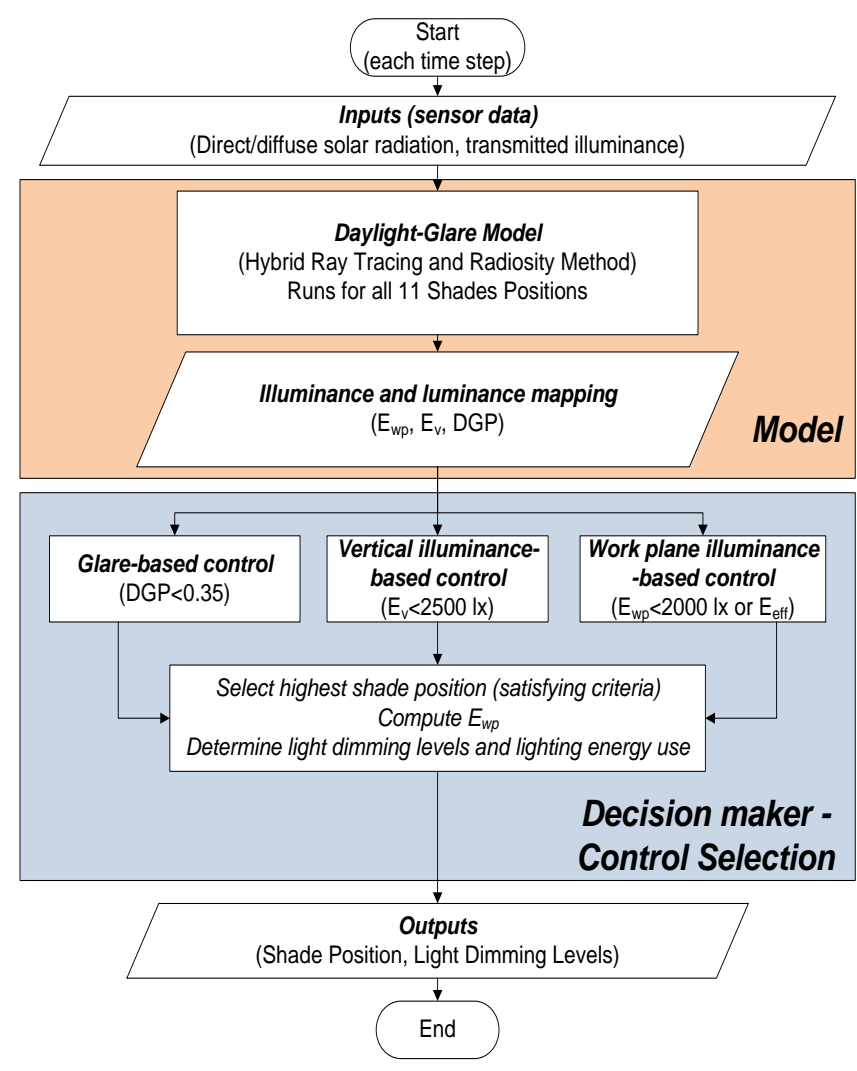

Figure 1. Flowchart of the developed model-based shading and lighting control

\subsection{Control criteria and control decision maker}

Based on the model outputs, the decision maker selects an intermediate shade position that allows the maximum amount of daylight (and reducing lighting energy use) while maintaining visual comfort. This is simply the "highest" shading position (among the 11 modeled in each time step) that satisfies a selected visual comfort criterion. If all shading positions fail to pass the criterion, the shades would be left closed. This approach is similar to an optimization scheme for discrete shade positions, using visual comfort constraints as a priority, but here it is applied efficiently with a semi-analytical model in real-time control.

To provide some insights on comfort criteria and their relative performance towards this approach, three visual comfort-based shading control criteria are considered and compared in this study as shown in Fig. 1:

1. DGP-based control. The highest shading position for which DGP $\leq 0.35$ is selected at each time step. Daylight Glare Probability is calculated based on Eq. (1).

2. Vertical illuminance $\left(E_{v}\right)$-based control. Konstantzos et al. (2015) showed that DGPs, the simplified version of DGP, which depends only on vertical illuminance on the eye $\left(E_{v}\right)$, is appropriate to use for all cases except when the sun is visible by the occupant (direct light falls on the eye):

DGPs $=6.22 \times 10^{-5} E_{v}+0.184$

The contrast term of DGP is not part of the simplified equation, therefore luminance distributions do not need to be computed, increasing computational efficiency. Eq. (2) is used to derive the vertical illuminance criterion: DGPs equal to 0.35 corresponds to $E_{v}=2670 \mathrm{~lx}$. Adding a small safety factor, the highest shading position for which $E_{v} \leq 2500$ lux is selected in the model-based control at each time step (calculating direct and diffuse portions separately). Note that shades with noticeable openness transmit 
direct light which might reach the occupant -for these cases, the use of DGPs is only recommended when the sun is not within the field of view of the occupant (Konstantzos et al., 2015).

3. Work plane illuminance $\left(E_{w p}\right)$-based control (or "effective illuminance" control). The highest shading position for which work plane illuminance $\left(\mathrm{E}_{\mathrm{wp}}\right)$ is lower than $2000 \mathrm{~lx}$ is selected for each time step without any direct sunlight reaching the work plane. The 2000 lux on the work plane is used as an index to align with the upper limit of UDI (Nabil and Mardaljevic, 2006), supported also by other studies with human subjects. The effective illuminance control (Shen and Tzempelikos, 2014) is a simplified modelbased control that requires only one light sensor installed on the window (transmitted illuminance reading). It does not really go through the real-time daylighting simulation; instead, it uses pre-simulated annual results to determine the maximum transmitted illuminance through the window and the shade ("effective illuminance") corresponding to $\mathrm{E}_{\mathrm{wp}}=2000$ lux close to the façade. This set point is not fixed and it depends on the orientation, location, room geometry, and reference work plane position. The relationship between transmitted illuminance and work plane illuminance is complex and not linear -but it is possible to select a reliable threshold that assures work plane illuminance values below 2000 lux. For the space used for the control implementation, described next, the transmitted illuminance threshold is 6000 lux when the buffer zone (minimum distance between the façade and the occupant) is $0.5 \mathrm{~m}$ (Shen et al., 2015).

After the shading position is selected, the controller extracts the simulated work plane illuminance (on the calculation grid) corresponding to the selected shading position for that time step and electric lights are controlled based on a work plane illuminance set point (500 lx in this study). This can be easily achieved by mapping dimming levels and $E_{w p}$, which will differ depending on the space and lighting system configuration and lighting control scheme. Light dimming can be implemented locally (per fixture or row of fixtures) or, for smaller rooms, based on the average $E_{w p}$. Lighting energy use is then calculated from corresponding light dimming levels for each time step and the entire calculation and control selection is repeated in the next time step.

\section{Model-based control implementation and experimental evaluation}

\subsection{Experimental setup: space, façade and lighting systems}

Two identical, side-by-side full-scale test offices (Fig. 2), part of the Architectural Engineering Laboratories at Purdue University were used to implement the developed model-based control strategies. The offices are equipped with reconfigurable façade, shading and lighting systems for investigating the impact of façade design and control options on indoor environmental conditions and energy use. The offices are $5 \mathrm{~m}$ wide by $5.2 \mathrm{~m}$ deep by $3.4 \mathrm{~m}$ high, and have a south facing façade with $60 \%$ vision area (window-to-wall ratio).
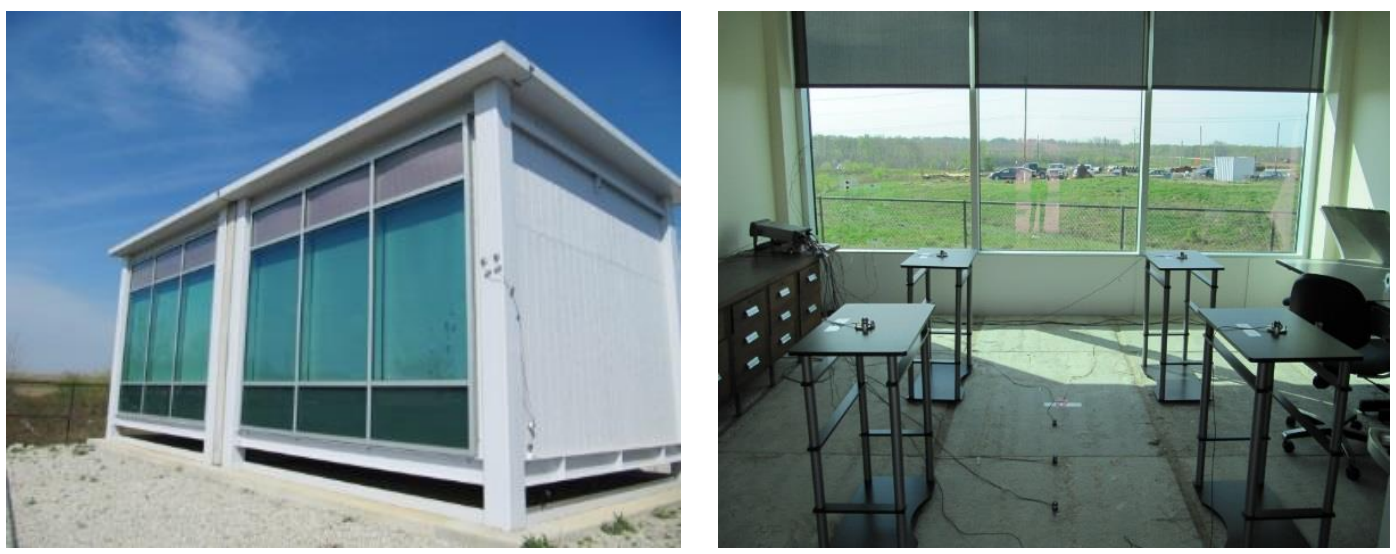

Figure 2. Exterior and interior view of the test offices 
The south facing façade consist of aluminum curtain wall framing (Kawneer 1600UT) and has a high performance glazing unit (SB70XL-clear with a spectrally selective low-emissivity coating, $2.1 \mathrm{~m}$ high). The glass detailed optical properties can be obtained from WINDOW 7 Software (normal visible transmittance $=65 \%$ ). The windows in both offices are equipped with motorized roller shades, with total visible transmittance equal to $5 \%$ (openness factor $=$ beam-beam transmittance at normal incidence $=$ $4.2 \%$ ), exterior reflectance equal to $74.5 \%$ and interior reflectance equal to $28 \%$ (grey color). The detailed angular optical properties of the fabrics used in the experiments were measured with an integrated sphere as described by Collins et al. (2012), and their angular properties (Fig. 3) were used in generalized modeling for simulation and MBC implementation. The beam-beam and beam-diffuse visible transmittance values are needed to be modeled separately, since direct and diffuse light transmission is required for the daylighting and glare simulation model. Note that the semi-empirical model by Kotey et al. (2009) was also validated and could be used in the absence of angular measurements. The diffuse-diffuse transmittance is computed by integrating the beam-total transmittance over the hemisphere.

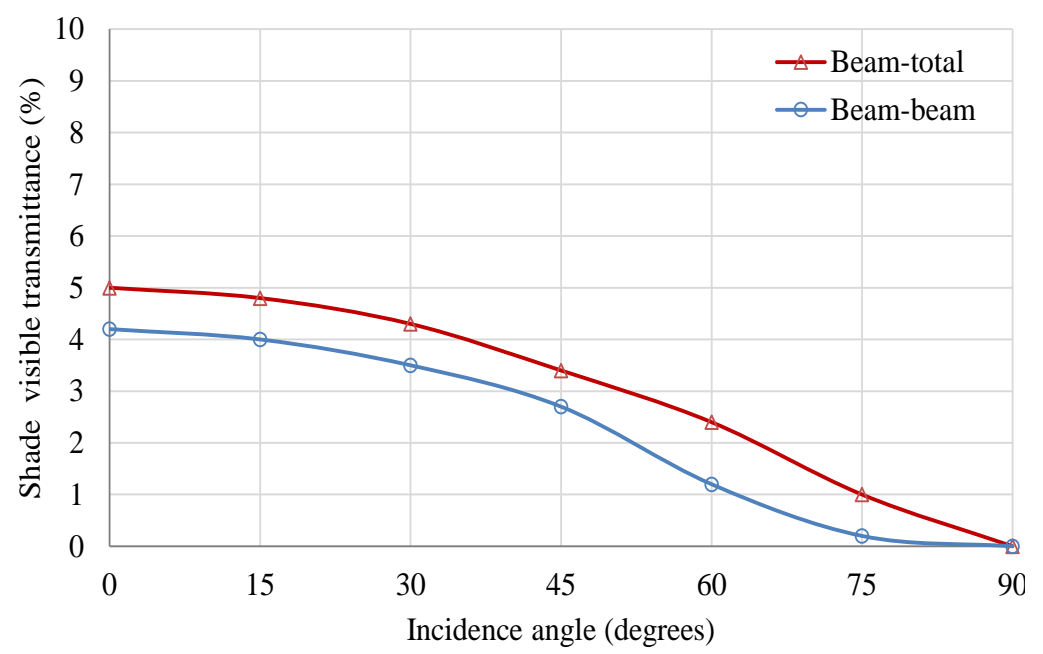

Figure 3. Detailed angular transmission properties of roller shades used in the experiments

The test offices are equipped with dimmable electric lighting systems that can be controlled according (in $1 \%$ increments) to any desired logic through acquiring and sending control signals to the ballasts. In each room, there are four light fixtures (two rows parallel to windows) with two lamps in each fixture (54W, T-5 high output, $4500 \mathrm{~lm}$ ). In this study, electric lights are controlled as a group to provide 500 lux on the work plane at all times (in larger rooms, lights should be controlled in rows to reduce energy use). After the decision maker selects the shade position, the calculated work plane daylight illuminance distribution is a matrix corresponding to a work plane grid in model ( $5 \times 5$ grid in this study). The average work plane illuminance value over the grid area is computed and the grid points that have illuminance values lower than 500 lux are used to determine the required electric light supplement from:

$$
{\overline{E_{w p}}}_{\text {demand }}=\frac{\sum E_{w p i<500 l x} \cdot\left(500-E_{w p i}\right)}{\operatorname{Grid} \#}
$$

where $E w p_{i}$ is daylight work plane illuminance of grid $i$ and Grid\# is the total number of grids in the daylight model (25 in this case). Mapping light dimming levels with average work plane illuminance without the presence of daylight is usually a straightforward process. In our case, where lights are 
controlled as a group, a simple power regression (Eq. 4) provides accurate results (Fig. 4). This will differ depending on the space geometry, lighting system configuration and control scheme.

$$
\text { Light dimming levels }=8 \times 10^{-6} \cdot{\overline{E_{w p}}}^{1.818}
$$

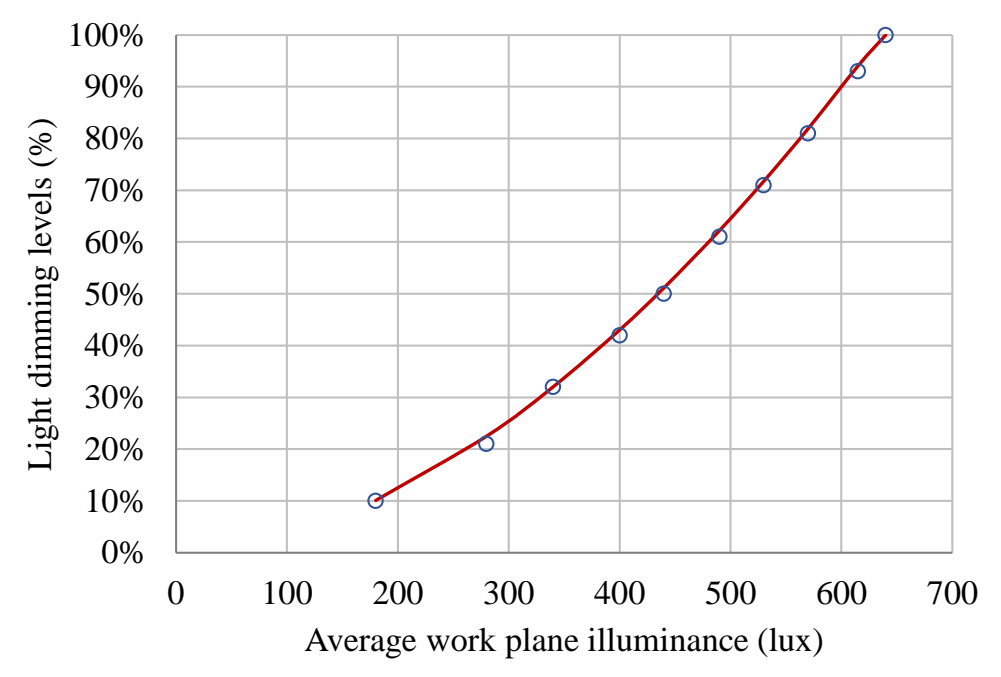

Figure 4. Light dimming levels correlated with average $E_{w p}$

\subsection{Instrumentation and sensor network}

Several LICOR calibrated photometers were used to measure light levels, both exterior (horizontal) and interior (transmitted illuminance through window, four horizontal work plane illuminances at $0.8 \mathrm{~m}$ height (Fig. 2), and vertical illuminance at the eye height level and at $2.20 \mathrm{~m}$ from the window). The light sensors have a cosine correction for incidence angles up to $80^{\circ}$, a response time of $0.01 \mathrm{~ms}$ and an absolute error of 3\%. Direct and diffuse incident solar radiation on the façade was measured by a SPN1 solar pyranometer, mounted on the exterior south wall. It is an effective alternative to a shade-ring pyranometer, designed to measure total and diffuse solar radiation simultaneously by using an array of seven thermopile sensors and a specially-designed shading mask (Fig. 5).

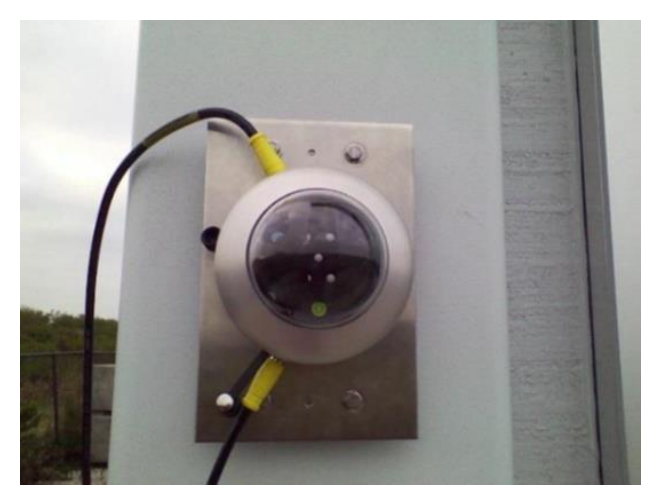

Figure 5. The SPN1 pyranometer used for measuring incident direct and total solar radiation on the façade

A calibrated Canon 550D dSLR camera equipped with a Sigma $4.5 \mathrm{~mm}$ fisheye lens, was used for validating luminance mapping and glare measurements. The camera was located in the center of the room 
(at a distance of $2.20 \mathrm{~m}$ from the facade), at the eye height level (Fig. 6). The calibration data was implemented in Labsoft v14.3.6 (Technoteam 2015), which was used for HDR creation, image processing and DGP calculation following the logic of Evalglare (Wienold, 2012). These measurements were used for model validation and overall control performance evaluation. Automated camera operation was enabled through a programmed mouse macro (Brothers Software 2015) controlling supportive software (EOS Utility), connected to the camera via USB cable. One set of three raw images with different exposures was taken by the mouse macro after a preset period (15 minutes in this study) and stored on the computer. Every set of three images are processed in Labsoft to create a luminance image based on calibration, and the luminance image was then processed to calculate a DGP value (Fig. 6). The photometer measuring vertical illuminance was placed right on top of the camera for validation purposes.

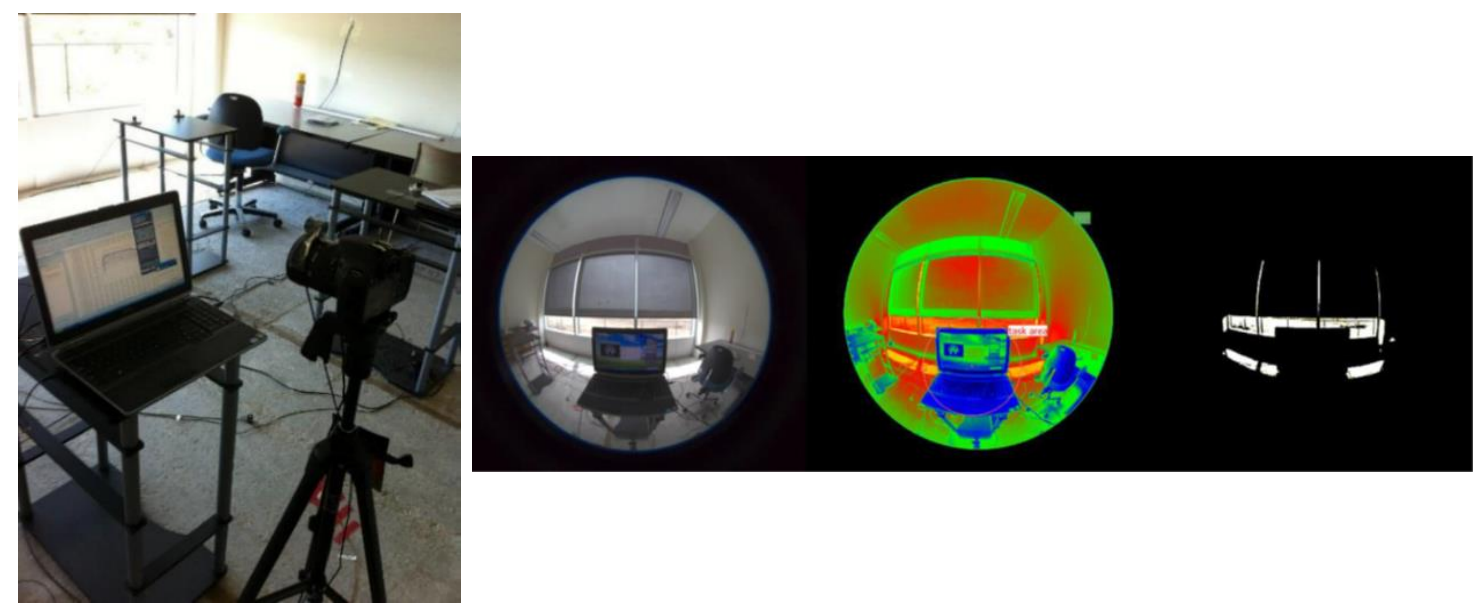

Figure 6. dSLR camera used for luminance mapping (left) and image processing using Labsoft to identify potential glare sources and estimate DGP values (right).

\subsection{Data acquisition and control framework}

The data acquisition and control hardware consist of a laptop, a control processor and a data acquisition/control unit. Agilent Technologies 34972A data acquisition units were used to combine precise measurements with flexible signal inputs. The control platform is a combination of Matlab v8.5 (R2014b) and LabVIEW Software (2013). The SPN1 pyranometer and the camera are directly connected to the laptop through USB port. Data measurements and controls are handled by LabVIEW, while the daylighting-glare model runs in Matlab. By utilizing the LabVIEW MathScript RT Module, the program is able to provide inputs to the Matlab model, execute the model in real time, and acquire the outputs. Control commands for shades and lights are sent to respective devices using Ethernet connections (Fig. 7). More specifically, in each control time loop ( $1 \mathrm{~min})$, the model-based control procedure is carried out as follows:

- LabVIEW program communicates with Agilent units and SPN1 pyranometer to obtain sensor readings;

- Sensor readings are processed, stored and sent to the Matlab daylighting model through MathScript;

- The main model (.m file) runs and the outputs are sent back to LabVIEW main program and stored;

- Commands for controlling shades and lights are generated from the control decision maker based on the model outputs; LabVIEW sends them through Ethernet to the control processor for adjustment of shade position and/or light dimming levels.

The entire process, summarized in Fig. 1, takes less than one second, which allows efficient real-time control considering all daylighting and glare calculations for the 11 shade positions at each time step. 


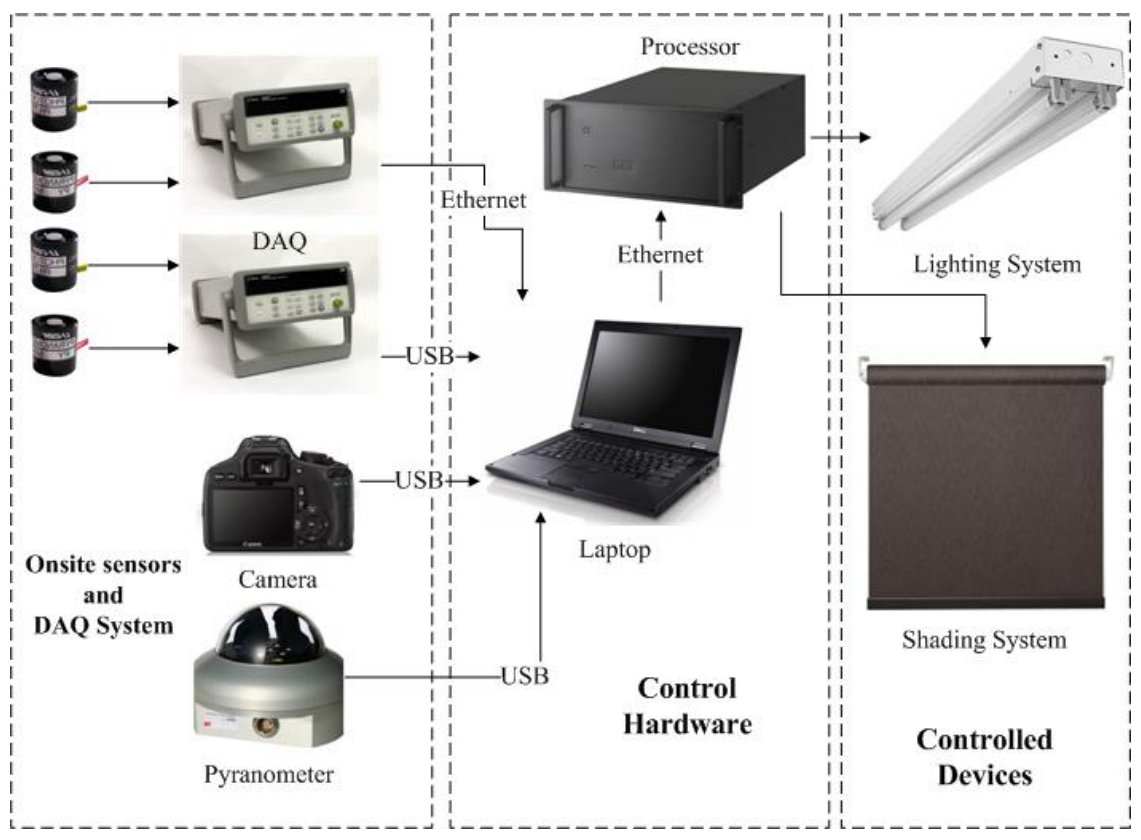

Figure 7. Components and connections of data acquisition and control hardware

\subsection{Implementation and comparison of model-based control strategies}

The three MBC strategies were implemented in the test offices during February-April 2015 (winter and spring), under a variety of sky conditions. They were simultaneously compared, with 1-min control interval, using real-time sensor measurements as input data, to evaluate their actual ability to maintain satisfactory visual comfort and minimize lighting energy use, and estimate their potential differences.

Representative results for three successive days with different sky conditions - one cloudy, one mixed and one sunny - are shown in Fig. 8. The comparison includes shading operation, indoor illuminances, light dimming levels and DGP, to validate the efficiency of the controls. Overall, all three MBC strategies were successfully implemented, achieving their visual comfort objectives respectively, while sufficient daylight was provided and electric light levels remained very low. The controls respond fast to changing conditions as shades move frequently during turbulent conditions (Fig. 8b). Outside view is also maintained since the shades do not close more than $70 \%$ even under the brightest conditions. Daylight provision is sufficient as work plane daylight illuminance remains between 500-2000 lux for most of the time (Fig. 8e). Electric light levels are minimal (below 20\% even for the first cloudy day in Fig. 8d) and thus consumed power remains low while sufficient daylight is provided, resulting in significant energy savings.

Considering visual comfort details (Fig. 8c), DGP- and $E_{v}$-based controls result in similar DGP conditions -DGP levels are kept under 0.35 all the time, even in partly cloudy days when shading movement is frequent due to varying outdoor lighting conditions. The $E_{v}$-based control seems to be a stricter criterion than the DGP-based control, since resulting vertical and work plane illuminances are lower. Evident differences between the work plane illuminance control and the other two strategies were observed during days with mixed and sunny sky conditions $\left(2^{\text {nd }}\right.$ and $3^{\text {rd }}$ day $) . E_{w p}$-based control results in lower shading fractions, consequently higher DGP values (up to 0.4) and higher vertical illuminance values (up to $3500 \mathrm{~lx}$ ), indicating the potential risk of glare. 

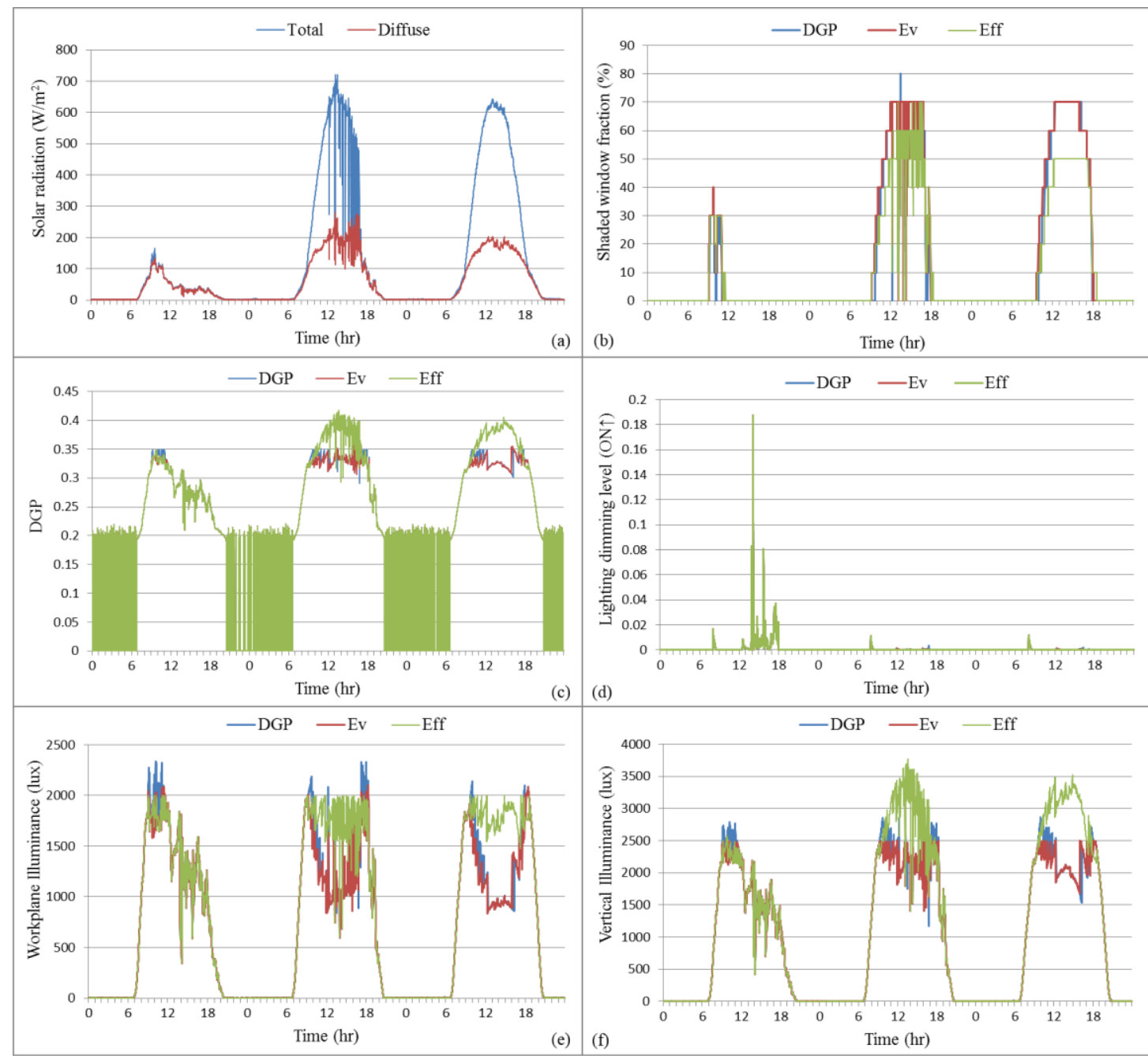

Figure 8. Implementation and experimental comparison of the three MBC strategies: (a) incident solar radiation on the façade; (b) recorded shading positions/fraction of outside view; (c) camera-measured $D G P ;(d)$ measured light dimming levels; (e) measured $E_{w p}$; and $(f)$ measured $E_{v}$

The $E_{v}$-based control performs well when the sun is not visible, and the differences with the full DGPbased control are small as expected. However, the effect of the contrast term on the controls is noticeable when there are bright areas within the field of view. An example of how this affects the MBC and the selected shading position is presented in Table 1. The luminance field, measured by the camera, is presented with the $E_{v}$ and the DGP-based controls for two instances in the same day in March-morning and afternoon, along with measured shade position and DGP values. The sunlight is projected in different directions during the day due to the south orientation of the façade. The DGP-based control results in slightly lower shade position and slightly lower DGP values.

Table 1. Differences between DGP and Ev-based MBC strategies during a sunny day in March

\begin{tabular}{|l|l|}
\hline \multicolumn{2}{|c|}{10 am } \\
\hline Ev-based control & DGP-based control \\
\hline
\end{tabular}




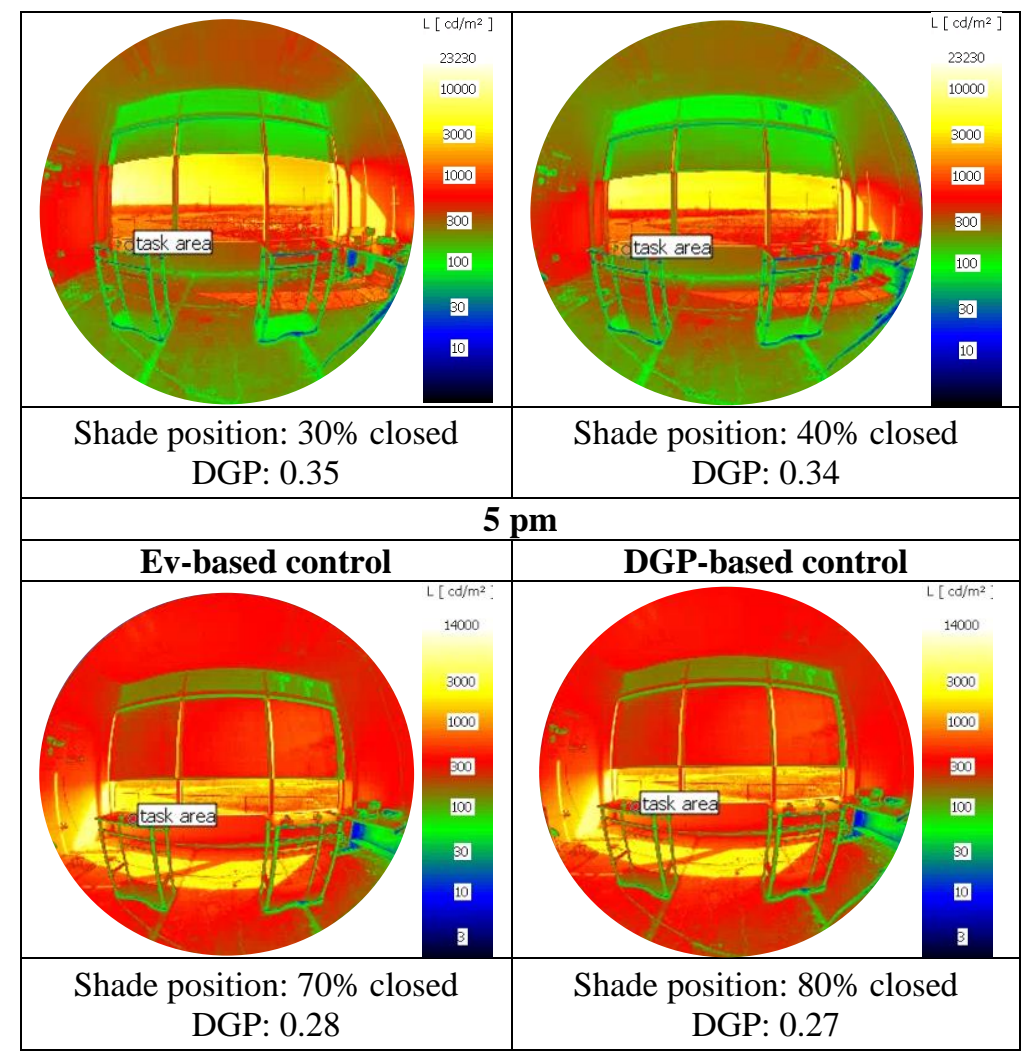

\section{Development and implementation of variable control interval MBC strategies}

The developed MBC strategies consider visual comfort as a priority and therefore require a fast response in shading and lighting operation under fluctuating sky conditions. To evaluate the efficiency of control response, the control interval was set to $1 \mathrm{~min}$ in the initial implementation. However, frequent shading and electric lighting operation could disrupt or disturb occupants and shorten the life of motors and lamps. Traditional control schemes try to improve this situation by "freezing" the controller for a specified period (e.g., 15 minutes). Although a freezing strategy could lower operation frequency, it may result in increasing risks of glare, especially during turbulent/mixed sky conditions. To prevent this, an advanced control logic was developed using variable control intervals, protecting from glare during mixed sky conditions (i.e., passing clouds) while reducing overall shading movement frequency.

The developed advanced variable interval logic flow chart is shown in Fig. 9. Measurements are still recorded every $1 \mathrm{~min}$ but control actions are decided separately. In each time step, the shade position (MSP), decided by the model-based control, is compared with the current shade position (CSP). If MSP is lower than CSP, which means shades need to lower for glare protection, control action is taken within a few seconds to move the shades to MSP, and the freezing timer is reset to 0. If MSP is higher than CSP and shades have not moved in the last 15 minutes (timer equals to 15), indicating that there was no required action for 15 minutes, shades would move to the minimum MSP calculated in the past freezing intervals, and the timer is reset to 0 ; otherwise shades should remain in the current position with the timer counting and the MSP of this time step is recorded for consideration in the next loop. This logic reduces the frequency of shade movements while allowing the maximum daylight according to the model-based control loop that prioritizes actions based on glare protection. 


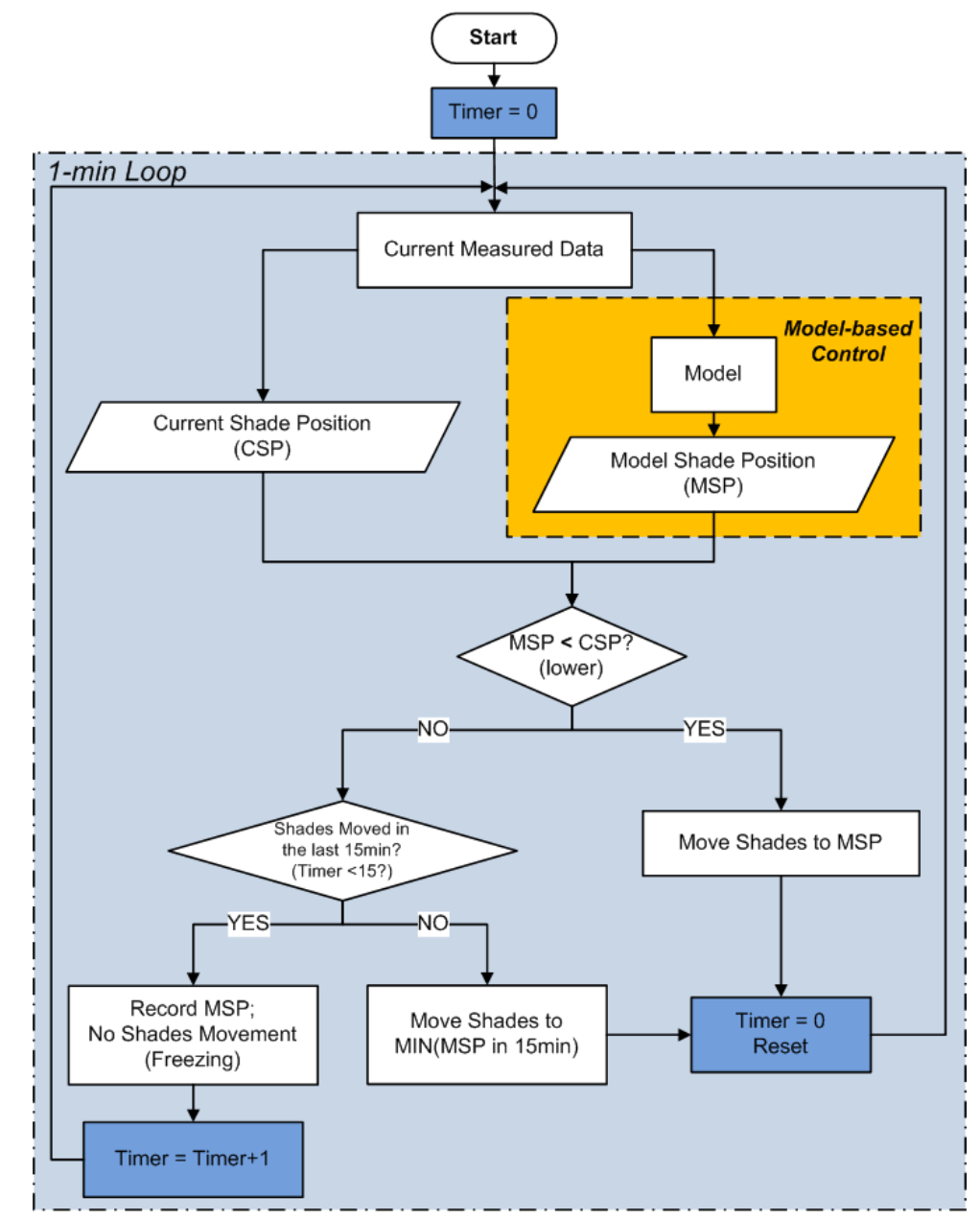

Figure 9. Flowchart of the advanced MBC variable interval logic for shading

The developed variable interval logic was implemented in the test offices, to evaluate its effectiveness and compare with the 1-min control operation implemented simultaneously (second office). Representative results for a day with turbulent sky conditions are shown in Fig. 10 for the DGP-based MBC option. The experimental results prove that the variable interval MBC can achieve the objective of reducing shade movements while maintaining visual comfort, without noticeable effects on lighting energy consumption. More specifically, the frequency of shade movement is significantly reduced with the variable control interval MBC (Fig. 10b), while visual comfort is well maintained (Fig. 10c), demonstrating the basic advantage of the variable control interval. The new logic reduces both work plane and vertical illuminances (Fig. 10e, 10f) mainly due to freezing actions when there is a predicted risk of glare. Nevertheless, indoor illuminances are sufficient for most of the time and therefore electric lighting levels are not significantly affected (Fig. 10d), which are considerably low during the day with both interval logics. 

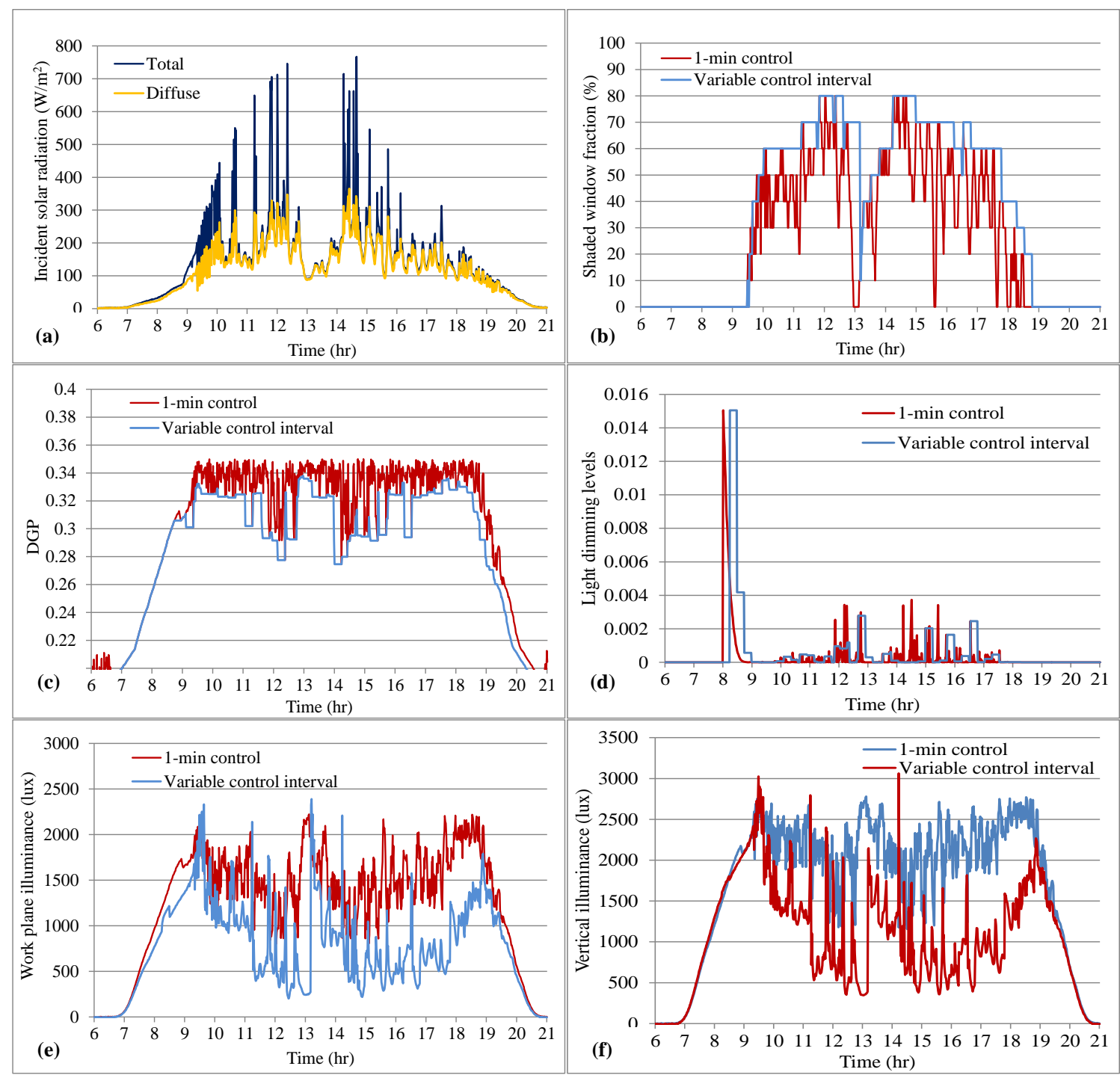

Figure 10. Shading MBC (DGP-based) results with 1-min interval and variable control interval during turbulent sky conditions: (a) incident solar radiation on the façade; $(b)$ recorded shading positions/fraction of outside view; (c) camera-measured DGP; $(d)$ measured light dimming levels; $(e)$ measured $E_{w p}$; and $(f)$ measured $E_{v}$

\section{Overall comparison of $\mathrm{MBC}$ strategies using annual simulation}

To generalize results and evaluate the overall performance of the developed MBC strategies, modelbased control of shading and lighting in the offices (same glazing and shade properties) was simulated for the entire year (using the 1-min control interval). Model inputs for every minute were obtained from linear interpolation of hourly TMY3 weather data for West Lafayette, Indiana. Although a linear interpolation of weather data cannot capture all the quick and turbulent changes in real outside conditions, the trend is satisfactory and simulation results can be safely used for a relative comparison of the different control strategies. 


\subsection{Comparison of annual performance of MBC strategies}

Annual shading operation schedules with the three controls are presented in Fig. 11. DGP and $E_{v}$-based strategies show similar shading operation for the entire year, while it is obvious that the effective control allows higher shade positions mainly during winter.
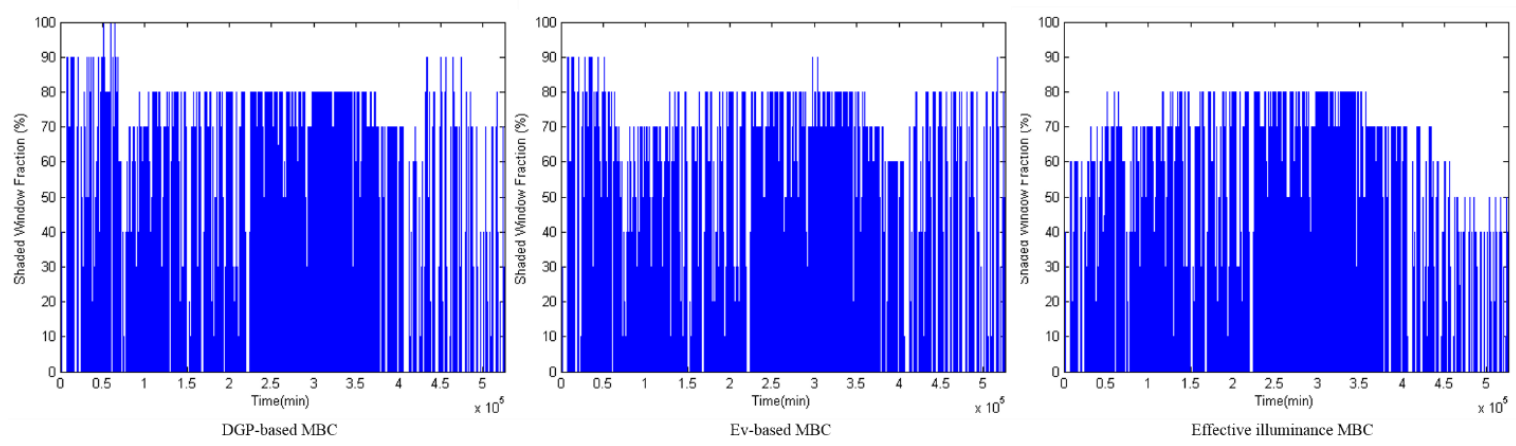

Figure 11. Annual shading operation with the three MBC strategies

Annual glare simulation results are shown in Fig. 12. In sunny winter days, all three strategies result in disturbing or intolerable glare (DGP>0.35), mainly due to direct sunlight transmitted through the fabric, reaching the occupant. Fabrics with smaller openness factor and visible transmittance should be used for south-facing orientations, and a method for selecting shade properties to reduce the risk of glare has been proposed by Chan et al. (2015). During the rest of the year, DGP-based control ensures DGP values below 0.35 and $E_{v}$-based control results in similar and slightly higher values (but below 0.4, which is the "intolerable" level according to the original DGP study). Model-based control using work plane illuminance as a criterion might lead to glare problems, since DGP values sometimes exceed 0.4 even in the summer, due to higher shade positions and higher vertical illuminance values.
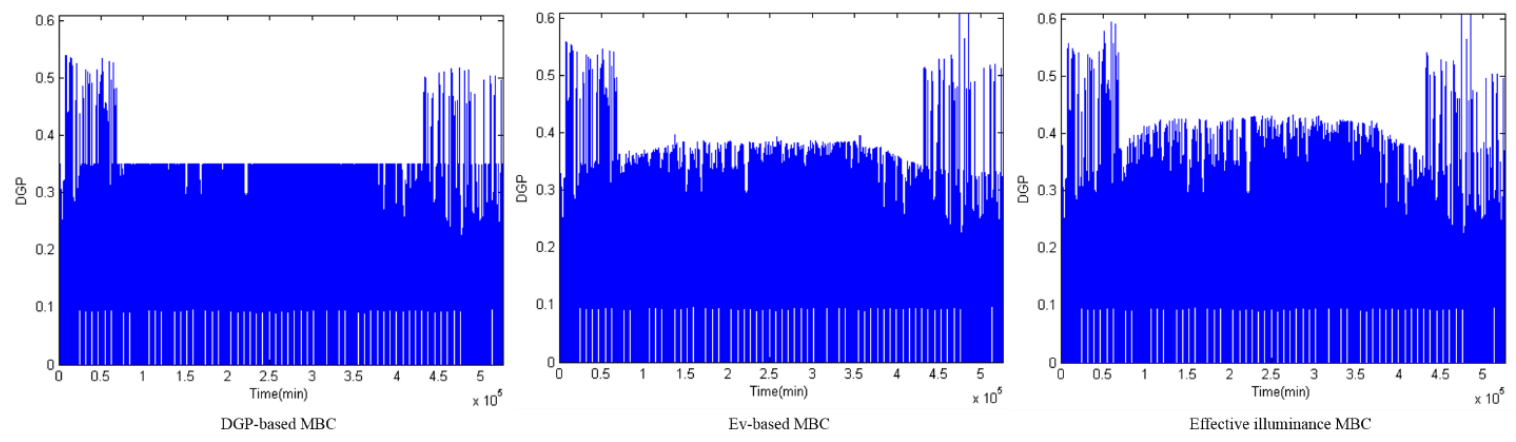

Figure 12. Annual DGP variation with the three MBC strategies

An overall comparison of the performance of the three MBC strategies is presented in Table 2. The DGP-based control results in lower frequency of shade movements and smaller percentage of time with noticeable glare. The effective illuminance ( $E_{w p}$-based) control could result in potential glare problems for a significant amount of time (considering the large windows and relatively high fabric openness).

The annual lighting energy consumption was also calculated based on the power capacity of the lamps used in the offices. Although DGP and $E_{v}$-based MBCs are stricter than the effective illuminance control (for glare protection), the energy use differences are negligible due to the high WWR and efficiency of controls. For cases with smaller windows, glazings with smaller visible transmittance, and other orientations, the lighting energy use will be considerably higher and the differences between the three 
strategies would be more evident. Nevertheless, the lighting energy savings are still expected to be quite high.

Table 2. Comparison of annual performance of the three MBC strategies (1-min control interval)

\begin{tabular}{c|c|c|c}
\hline & DGP-based MBC & $E_{v}$-based MBC & $\begin{array}{c}\text { Effective illuminance } \\
\left(E_{w p} \text {-based) control }\right.\end{array}$ \\
\hline Count of shade movements & 4964 & 5209 & 5190 \\
\hline $\begin{array}{c}\text { Average shaded window } \\
\text { fraction (\%) }\end{array}$ & 23.4 & 23.5 & 22.8 \\
\hline $\begin{array}{c}\text { Time percentage (working } \\
\text { hour) when DGP>0.35 }\end{array}$ & $2.25 \%$ & $7.5 \%$ & $22.59 \%$ \\
\hline $\begin{array}{c}\text { Annual lighting energy } \\
\text { consumption (kWh) }\end{array}$ & 57.1 & 58.3 & 55.0 \\
\hline
\end{tabular}

\subsection{Overall evaluation of variable control interval MBC using annual simulation}

The performance of MBC with the variable control interval logic was also evaluated using annual simulation results, for the case of DGP-based control. There are no significant differences in the resulting DGP values between the variable interval and the frequent (1-min) controls, as shown in Fig. 13 - the time percentage with noticeable glare also remains the same. However, the shading movement frequency is reduced by almost $25 \%$ (Table 3). This difference might be even higher in real-time control, since linear interpolation of weather data create "smooth" input simulation data for the 1-min control interval. Overall, the developed MBC control with variable control interval reduces the frequency of shading operation while satisfying glare constraints and allowing adequate daylight.

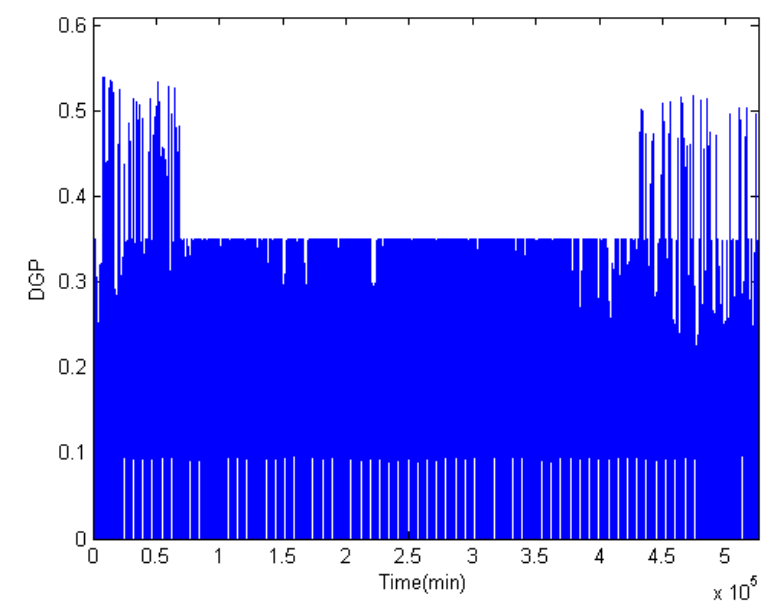

Figure 13. Annual DGP variation with the DGP-based MBC strategy with variable control interval

Table 3. Frequency of annual shade movements using the DGP-based MBC with 1-min and variable control intervals

\begin{tabular}{l|l|l}
\hline & 1 -min control interval & Variable control interval \\
\hline
\end{tabular}




\begin{tabular}{l|l|l}
\hline Count of shade movements & 4964 & 3794 \\
\hline
\end{tabular}

\section{Conclusion}

This study presents the development and implementation of model-based shading and lighting control algorithms, aiming at minimizing lighting energy use while maintaining visual comfort and adequate daylight levels using a small number of sensors. The developed model and controls can decrease sensor network complexity while retaining reliability.

Three control criteria, based on DGP, vertical and horizontal (effective) illuminance were evaluated and compared. A fast and efficient daylighting and glare model was used to compute all required quantities for predetermined shade positions ( $10 \%$ increments) at each time step. The control logic selects the highest shade position at each time step that satisfies the visual comfort criteria, to maximize daylight utilization and minimize lighting energy use.

The control strategies were successfully implemented in full-scale test offices with satisfactory results. The real-time model-based control is able to capture rapidly changing sky conditions and take appropriate action. The experimental results showed that DGP values remained below 0.35 for most cases while work plane illuminance levels were adequate, and lighting energy use was significantly reduced with all MBC strategies. Work plane illuminance control resulted in higher shade positions with potential risk of glare in some cases.

An advanced, variable control interval logic was also developed, aiming to reduce the frequency of shade movements while still achieving high lighting energy savings and maintaining glare protection and outside view. The variable control MBC was also successfully implemented in the offices, resulting in reduced shading operation without sacrificing the benefits, which improves the feasibility of practical applications, less disturbance for occupants and extension of equipment life.

The developed MBC strategies were embedded in annual simulation to evaluate their overall performance. The DGP-based control proved to be the most efficient; nevertheless, model-based control based on vertical illuminance, which is easier to calculate and more practical, shows similar benefits. When the sun is within the field of view, fabrics of small openness are required to reduce the risk of glare -for higher openness factors and transmittance, the vertical illuminance criterion should not be sufficient for ensuring visual comfort.

This work is an initial step for developing integrated model-based controls for perimeter zones of commercial buildings. Future work includes applying and testing the developed MBCs in occupied offices, to evaluate human satisfaction with the developed controls and interactions with shading and lighting systems. Also, simultaneous consideration of the thermal environment in the controls, combined with model-predictive approaches, will allow integrated and coordinated systems control with required intelligence embedded in the building cyber infrastructure.

\section{Acknowledgments}

This work was partially funded by the National Science Foundation under Grant No. 1539527 (any opinions, findings, and conclusions or recommendations expressed in this material are those of the authors and do not necessarily reflect the views of the National Science Foundation). The authors would like to thank Lutron Electronics Co Inc and Kawneer Inc for providing the experimental infrastructure (reconfigurable façade, shading and lighting systems and controls). Thanks also Dr. Ying-Chieh Chan for helping with the model development and Jason Konstantzos for assisting with the camera measurements.

\section{References}

Brothers Software. Quick Macro 6.6. http://www.qmacro.com/ 
Bueno, B., Wienold, J., Katsifaraki, A., Kuhn, T.E. Fener: A Radiance-based modelling approach to assess the thermal and daylighting performance of complex fenestration systems in office spaces. Energy and Buildings 94, 10-20.

Cai, J., Braun, J.E., 2015. A generalized control heuristic and simplified model predictive control strategy for direct-expansion air-conditioning systems. Science and Technology for the Built Environment 21 (6), 773-778.

Chan, Y. C., Tzempelikos, A., Konstantzos, I., 2015. A systematic method for selecting roller shade properties for glare protection. Energy and Buildings, 92, 81-94.

Chan, Y-C., Tzempelikos, A., 2012. A hybrid ray-tracing and radiosity method for calculating radiation transport and illuminance distribution in spaces with venetian blinds. Solar Energy 86(11), 3109-3124.

Daum, D., Morel, N., 2010. Identifying important state variables for a blind controller. Building and Environment, 45, 887-900.

Derakhtenjani, A.S., Candanedo, J.A., Chen, Y., Dehkordi, V.R., Athienitis, A.K., 2015. Modeling approaches for the characterization of building thermal dynamics and model-based control: a case study. Science and Technology for the Built Environment 21 (6), 824-836.

EIA, 2015. US Energy Information Administration: Energy Consumption by Sector and Source. Annual Energy Outlook 2015.

Fisher, M., Wu, K., Agathoklis, P., 2012. Intelligent Illumination Model-Based Lighting Control. Proceedings of 32nd International Conference on Distributed Computing Systems Workshops, Macau.

Gruber, M., Trüschel, A., Dalenbäck, J-O., 2014. Model-based controllers for indoor climate control in office buildings-complexity and performance evaluation. Energy and buildings 68, 213-222.

Guillemin, A., Molteni, S., 2002. An energy-efficient controller for shading devices self-adapting to the user wishes. Building and Environment, 37, 1091-1097.

Guth, S. K., 1966. Computing visual comfort ratings for a specific interior lighting installation. Illuminating Engineering, 61(10), 634-642.

Hu, J., Karava, P., 2014. A state-space modeling approach and multi-level optimization algorithm for predictive control of multi-zone buildings with mixed-mode cooling. Building and Environment 80, 259-273.

Inoue, T., Kawase, T., Ibamoto, T., Takakusa, S., Matsuo, Y., 1988. The development of an optimal control system for window shading devices based on investigations in office buildings. ASHRAE transactions 94, 1034-1049.

Kim, D.W., Park, C.S., 2012. Comparative control strategies of exterior and interior blind systems. Lighting Research and Technology 44, 291-308.

Konstantzos, I., Tzempelikos, A., Chan, Y-C., 2015. Experimental and simulation analysis of daylight glare probability in offices with dynamic window shades. Building and Environment 87, 244-254.

Kotey, N.A., Wright, J., Collins, M.R., 2009. Determining off-normal solar optical properties of roller blinds. ASHRAE Transactions 117 (1).

LBNL, 2015. WINDOW 7.3 software. Lawrence Berkeley National Laboratory. Available from: https://windows.lbl.gov/software/window/window.html

Le, K., Bourdais, R.,Guéguen, H., 2014. From hybrid model predictive control to logical control for shading system: a support vector machine approach. Energy and Buildings 84, 352-359.

Li, S., Joe, J., Hu, J., Karava, P., 2014. System identification and model-predictive control of office buildings with integrated photovoltaic-thermal collectors, radiant floor heating and active thermal storage. Solar Energy 113, 139-157.

Lindelof, D., 2009. A fast daylight model suitable for embedded controllers. Solar Energy 83, 57-68. 
Lu, T., Lü, X., Viljanen, M., 2011. A novel and dynamic demand-controlled ventilation strategy for CO 2 control and energy saving in buildings. Energy and buildings 43 (9), 2499-2508.

Mahdavi, A. Predictive simulation-based lighting and shading systems control in buildings. Building Simulation 1(1), 25-35.

Moroşan, P-D., Bourdais, R., Dumur, D., Buisson, J., 2010. Building temperature regulation using a distributed model predictive control. Energy and Buildings 42 (9), 1445-1452.

Nabil, A., Mardaljevic, J., 2006. Useful daylight illuminances: a replacement for daylight factors. Energy and Buildings 38 (7), 905-913.

Oh, M.H., Lee, K.H., Yoon, J.H., 2012. Automated control strategies of inside slat-type blind considering visual comfort and building energy performance. Energy and Buildings 55, 728-737.

Pengfei, L., Vrabie, D., Li, D., Bengea, S.C., Mijanovic, S., O’Neill, Z.D., 2015. Simulation and experimental demonstration of model predictive control in a building HVAC system. Science and Technology for the Built Environment 21 (6), 721-732.

Privara, Samuel, Siroky, J., Ferkl, L., Cigler, J., 2011. Model predictive control of a building heating system: The first experience. Energy and Buildings 43 (2), 564-572.

Shen, H., Hu, J., Patel, M., 2014. Energy and visual comfort analysis of lighting and daylight control strategies. Building and Environment 78, 155-170.

Shen, H., Tzempelikos, A., 2014. A global method for efficient synchronized shading control using the "effective daylight" concept. Proceedings of 3rd International High Performance Buildings Conference at Purdue, West Lafayette, Indiana, 8 pages.

Shen, H., Tzempelikos, A., Atzeri, A.M., Gasparella, A., Cappelletti, F., 2015. Dynamic commercial facades versus traditional construction: energy performance and comparative analysis. ASCE Journal of Energy Engineering, 141 (4), 04014041.

Technoteam, LMK Labsoft 14.3.6. Available from: http://www.technoteam.de/product_overview/lmk/software/lmk_labsoft/index_eng.html

Tzempelikos, A., Shen, H., 2013. Comparative control strategies for roller shades with respect to daylighting and energy performance. Building and Environment 67, 179-192.

Van Den Wymelenberg, K., Inanici, M., 2014. A Critical Investigation of Common Lighting Design Metrics for Predicting Human Visual Comfort in Offices with Daylight. LEUKOS 10 (3), 145-164.

Wienold, J., 2007. Dynamic simulation of blind control strategies for visual comfort and energy balance analysis. Proceedings of IBPSA 2007 Conference, Beijing, China, pp. 1197-1204.

Wienold, J., 2012. EvalGlare Version 1.0. Fraunhofer Institute for Solar Energy Systems, Freiburg.

Wienold, J., Christoffersen, J. Evaluation methods and development of a new glare prediction model for daylight environments with the use of CCD cameras. Energy and Buildings 38, 743-757.

Yun, G.Y., Yoon, K.C., Kim, K.S., 2014. The influence of shading control strategies on the visual comfort and energy demand of office buildings. Energy and Buildings 84, 70-85. 